Pacific Journal of Mathematics

WEAK COMPACTNESS AND SEPARATE CONTINUITY 


\section{WEAK COMPACTNESS AND SEPARATE CONTINUITY}

\section{IRVING GLICKSBERG}

1. For a locally compact space $X$ let $C(X)$ denote the Banach space of all bounded continuous complex valued functions on $X, C_{0}(X)$ the subspace of functions vanishing at infinity, so that the adjoint $C_{0}(X)^{*}$ consists of all finite complex regular Borel measures on $X$. In a natural fashion, we may view $C(X)$ as a subspace of $C_{0}(X)^{* *}$.

When $X$ is compact Grothendieck [6; Th. 5] has shown that a bounded set $K \subset C(X)$ is compact in the weak topology if (and of course only if) $K$ is compact in the topology of pointwise convergence on $X$, and then both topologies, being comparable, coincide on $K$. In some recent work the writer was led to a simple corollary of Grothendieck's result which yields the significance, when $X$ is only locally compact, of compactness in $C(X)$ under pointwise convergence:

1.1. Let $K$ be a bounded subset of $C(X), X$ locally compact. Then $K$ is compact in the topology of pointwise convergence on $X$ (if and). only if $K$ is compact in the weak* topology of $C_{0}(X)^{* *}[4,5.1]$.

Again both topologies coincide on $K$. A direct corollary of 1.1 is

1.2. Let $X$ and $Y$ be locally compact spaces, and $f$ a bounded complex function on $X \times Y$ which is separately continuous, i.e., for which all the maps

$$
x \rightarrow f(x, y) \text { and } y \rightarrow f(x, y)
$$

are continuous. Then for $\mu \in C_{0}(X)^{*}$,

$$
y \rightarrow \int f(x, y) \mu(d x)
$$

is continuous [4, 5.2].

The continuity obtained in 1.2 allows one to form the iterated integral

$$
\iint f(x, y) \mu(d x) \nu(d y), \quad \mu \in C_{0}(X)^{*}, \nu \in C_{0}(Y)^{*},
$$

and thus one can extend the notion of convolution of a pair of finite measures to a locally compact semigroup $S$ in which the operation is only separately continuous. Moreover 1.2 shows (1.21) is identical with

Received March 24, 1960. 


$$
\iint f(x, y) \nu(d y) \mu(d x)
$$

so that convolution is commutative if $S$ is. Consequently we show in $\S 4$ how some results of [3] extend to the separately continuous situation; these in turn yield an analogue of the Weyl equidistribution theorem which applies to weakly almost periodic functions on locally compact abelian groups (4.6 below).

Although the fact will not be needed in what follows, note that 1.1 is actually a weak compactness criterion for the complete locally convex space $C(X)_{\beta}$ formed from $C(X)$ by endowing it with the strict topology (cf. [o]). For since the dual $C(X)_{\beta}^{*}$ consists precisely of the measures in $C_{0}(X)^{*}$, the weak topology of $C(X)_{\beta}$ is just the weak* topology in 1.1 and the bounded sets of $C(X)$ and $C(X)_{\beta}$ coincide. But as a consequence the topology of pointwise convergence on $C(X)$, when restricted to bounded sets, shares some properties of weak topologies of complete locally convex spaces: conditionally countably compact sets are conditionally compact, and have compact convex hulls.

Notation. For a function $f, f \mid E$ will denote its restriction to $E$, while for a set $K$ of functions, $K \mid E$ will denote the corresponding set of restrictions. $C(X)_{p}$ and $C(X)_{w^{*}}$ will denote $C(X)$ in the topology of pointwise convergence on $X$, and in the weak* topology of $C_{0}(X)^{* *}$, respectively. In general $X$ and $Y$ will denote locally compact (Hausdorff) spaces, and, for a function $f$ on $X \times Y, f(\cdot, y)$ will be its section $x \rightarrow f(x, y)$ (with $f(x, \cdot)$ defined analogously). As we have indicated $f$ is separately continuous only if all of sections are continuous. Other notation is standard.

2. Since the proofs of 1.1 and 1.2 (given in [4]), are quite short, we shall include them for completeness.

Consider 1.1, and let $\mathscr{F}$ be an ultrafilter on $K$. $\mathscr{F}$ converges to some $f_{0}$ in $K$ in $C(X)_{p}$, and we need only show $\mathscr{F}$ converges to $f_{0}$ in $C(X)_{w^{*}}$. On the bounded set $K$ the weak* topology is defined by the dense set of measures $\mu$ with compact carriers $C_{\mu}$, so we need only show $\int f_{0} d \mu=\lim _{\mathscr{F}} \int f d \mu$ for such $\mu$. But $K \mid C_{\mu}$ is compact in $C\left(C_{\mu}\right)_{p}$ and thus, by Grothendieck's theorem, compact in the weak topology, and both topologies coincide on $K \mid C_{\mu}$. Clearly then $\int f_{0} d \mu=\lim _{\mathscr{F}} \int f d \mu$ as desired.

In order to prove 1.2 we have to show the map $y \rightarrow f(\cdot, y)$ of $Y$ into $C(X)_{w^{*}}$ is continuous. But it is a continuous map into $C(X)_{p}$, so that any compact neighborhood $V$ of $y_{0} \in Y$ has an image which is compact in the weak* topology by 1.1. And since the weak* topology coincides on this image with that of pointwise convergence, the desired continuity is immediate. 
As a first application of 1.2 we note the following simple proof of the well known fact (due to Krein and Smulian) that if $K$ is a weakly compact subset of a complete locally convex linear space $E$, then the closed convex hull $\mathscr{C}(K)$ is weakly compact. Take, as our $X$ and $Y$ of 1.2, $K$ in the weak topology, and the polar $V^{0} \subset E^{*}$ of a neighborhood $V$ of 0 in $E$, in the weak* topology. Since $x \rightarrow\left\langle x, x^{*}\right\rangle$ and $x^{*} \rightarrow\left\langle x, x^{*}\right\rangle$ are each continuous in the appropriate topologies, by 1.2 we have, for $\mu \in C(K)^{*}$,

$$
x^{*} \rightarrow \int\left\langle x, x^{*}\right\rangle \mu(d x)
$$

continuous on $V^{0}$. Since $V$ is an arbitrary neighborhood of 0 , and $E$ is complete, a well known result of Grothendieck [5] shows (2.11) represents a weak* continuous functional on $E^{*}$, and thus there is an $x_{\mu}$ in $E$ satisfying

$$
\left\langle x_{\mu}, x^{*}\right\rangle=\int\left\langle x, x^{*}\right\rangle \mu(d x),
$$$$
x^{*} \in E^{*} \text {. }
$$

Let $N=\left\{\mu: \mu \in C(K)^{*}, \mu \geqq 0, \mu(K)=1\right\}$, a weak* compact convex subset of $C(K)^{*}$, and endow $N$ with the weak* topology. Since

$$
\mu \rightarrow \int\left\langle x, x^{*}\right\rangle \mu(d x)
$$

is clearly continuous on $N$, (2.12) implies $\mu \rightarrow x_{\mu}$ is a continuous map from $N$ into $E$ under the weak topology; thus the range of this map is a convex weakly compact subset of $E$, which clearly contains $K$. Since $\mathscr{C}(K)$ is weakly closed by Mazur's theorem, this is all we need to show.

3. As was noted in the introduction, 1.2 allows one to form the iterated integral

$$
\iint f(x, y) \mu(d x) \nu(d y), \quad \quad \mu \in C_{0}(X)^{*}, \nu \in C_{0}(Y)^{*},
$$

for any bounded separately continuous $f$. The desirable interchangability of the order of integration would of course be immediate once $f$ is, say, locally Borel measurable; however the writer is not aware of any general answer to the question of measurability of separately continuous functions (a special case is covered in [7, §39]). Nevertheless the independence of order is easily obtained from 1.2.

THEOREM 3.1. Let $f$ be a bounded separately continuous complex function on $X \times Y$. Then

$$
\iint f(x, y) \mu(d x) \nu(d y)=\iint f(x, y) \nu(d y) \mu(d x), \mu \in C_{0}(X)^{*}, \nu \in C_{0}(Y)^{*}
$$


Proof. Let $\mu$ be fixed. For $K$ a compact subset of $Y$ let $E_{K}=$ $\left\{\nu:\|\nu\| \leqq 1, \nu\right.$ vanishes on subsets of $\left.K^{\prime}\right\}$. Clearly (3.11) holds when $\nu$ is a finite linear combination of point masses; since these are weak ${ }^{*}$ dense in $E_{K}$ we can prove (3.11) holds for all $\nu$ in $E_{K}$ by showing both sides are continuous functions on $E_{K}$, taken in the weak* topology of $C_{0}(Y)^{*}$. By Urysohn's lemma this topology coincides on $E_{K}$ with the weak* topology of $C(K)^{*}$, and thus the left side of (3.11) is continuous since the inner integral represents an element of $C(K)$. On the other hand $E_{K}$ is compact in the weak* topology of $C(K)^{*}$ and

$$
(x, \nu) \rightarrow \int f(x, y) \nu(d y)
$$

defines a bounded separately continuous function on $X \times E_{K}$ (by 1.2 and the definition of the weak* topology). Thus 1.2 implies

$$
\nu \rightarrow \iint f(x, y) \nu(d y) \mu(d x)
$$

is continuous on $E_{K}$.

Consequently (3.11) holds for any given $\mu$, and any $\nu$ with compact carrier. Since such $\nu$ are strongly dense in $C_{0}(X)^{*},(3.11)$ follows.

4. Let $S$ be a compact space which is also a semigroup (group), and suppose the operation is separately continuous:

$$
x \rightarrow x y \text { and } y \rightarrow x y
$$

are continuous; then we shall call $S$ a compact separately continuous semigroup (group). For $\mu$ and $\nu$ in $C(S)^{*}$ we can form the convolution of $\mu$ and $\nu$, an element $\mu \nu$ of $C(S)^{*}$, by virtue of the Riesz representation theorem and 1.2:

$$
\int f(x) \mu \nu(d y)=\iint f(x y) \mu(d x) \nu(d y), \quad f \in C(S) .
$$

Convolution is easily seen to be associative, and endowing $C(S)^{*}$ with its weak* topology, separately continuous (by 3.1). Moreover 3.1 shows. convolution is commutative when $S$ is.

Let $\widetilde{S}=\left\{\mu: \mu \in C(S)^{*}, \mu \geqq 0, \mu(S)=1\right\} ; \widetilde{S}$ forms a compact separately continuous semigroup under convolution and the weak* topology. In [3] the writer determined the subgroups of $\widetilde{S}$ when $S$ is also jointly continuous; in the present section we shall see how some of the results. of [3] extend to the separately continuous situation. (We might remark that compact separately continuous semigroups arise naturally in the study of weakly almost periodic functions on, for example, the real line (cf. [2])). 
That most of these results carry over to the separately continuous situation is due to the consequences of Grothendieck's theorem given above. We shall also make mild use ${ }^{1}$ of a fact due to Ellis [1] which can be obtained, interestingly enough, from Grothendieck's result [2, Appendix]: a compact separately continuous group is a compact topological group. In particular any closed algebraic subgroup of $S$ is a compact topological group. (However an algebraic subgroup need not have its closure an algebraic subgroup, as in the jointly continuous case.)

To begin, let us note some distinctions between the present, separately continuous, situation, and that of [3], preserving, insofar as possible, the notation of [3]. When $S$ is separately continuous, only the same is true of $\widetilde{S}$ in general. But all of the ideal structure used in [3] continues to hold (with one exception: (1.11) of [3] fails); in particular every abelian separately continuous compact semi-group $S$ contains a least ideal $\left(\bigcap_{x \in S} x S\right)$ which is closed, a group, and thus a compact topological group. (In [3] we allowed $S$ to be abelian, or a group; by virtue of the result cited above nothing new is obtained by allowing $S$ to be a group here, and we shall insist that $S$ be abelian in all but our first result.) The following is, in modified form, the key lemma of [3].

Lemma 4.1. Let $S$ be a compact separately continuous semi-group, and let $\mu, \nu \in \widetilde{S}$. Then

$$
\text { carrier } \mu \nu=[(\text { carrier } \mu)(\text { carrier } \nu)]^{-} \text {. }
$$

Proof. The proof given in [3, Lemma 2.1] with $A \cdot B$ replaced by the right side of (4.11) shows the right side has $\mu \nu$-measure 1 . To see that any open set $W$ which meets the right side of (4.11) has $\mu \nu(W)>0$, we argue as follows.

Let $x_{0} y_{0} \in W, x_{0} \in$ carrier $\mu, y_{0} \in$ carrier $\nu$. Then if $f \in C(S)$ vanishes off $W$ while $f\left(x_{0} y_{0}\right)=1,0 \leqq f \leqq 1$, we have $\int f\left(x y_{0}\right) \mu(d x)>0$ since $x \rightarrow f\left(x y_{0}\right)$ is positive near $x=x_{0}$. Since $y \rightarrow \int f(x y) \mu(d x)$ is continuous by 1.2 , and positive at $y=y_{0}$,

$$
0<\iint f(x y) \mu(d x) \nu(d y)=\int f(z) \mu \nu(d z) \leqq \mu \nu(W) .
$$

Consequently the right side of (4.11) is indeed carrier $\mu \nu$.

In the remainder of this section we assume that $S$ is an abelian compact separately continuous semigroup.

${ }^{1}$ Essentially we use this to assert that $\mu$ in 4.2 , when shown to be an invariant normalized measure on a separately continuous compact group, is the Haar measure; of course this could easily be avoided. 
THEOREM 4.2. Let $\mu^{2}=\mu \in \widetilde{S}$. Then carrier $\mu$ is a compact subgroup of $S$, and $\mu$ its Haar measure.

If $H=$ carrier $\mu$, then 4.1 shows $H^{2-}=H$, and scrutiny of the proof of [3, Th. 2.2] shows this is an adequate replacement for $H^{2}=H$. (Note that 1.2 must be used to obtain the continuity of $f^{\prime}$.)

THeOREM 4.3. Let $\Gamma$ be an algebraic subgroup of $\widetilde{S}$. Then $G=\bigcup_{\mu \in \Gamma}$ carrier $\mu$ is an algebraic subgroup of $S$. If $\eta$ is the identity of $\Gamma, g=$ carrier $\eta$ is a compact topological group, $\eta$ its Haar measure, and $\Gamma$ the set of $G$-translates of $\eta$. Furthermore if $\Gamma$ is closed, $G$ is closed.

Proof. $G$ is algebraically a subsemigroup of $S$ by 4.1 , while $g$ is a compact group and $\eta$ its Haar measure by 4.2. Let $e$ be the identity of $g$. Then for $\mu \in \Gamma, x \in$ carrier $\mu=[g \text { carrier } \mu]^{-}$implies $e x=x$ since this holds for $x$ in $g$ carrier $\mu$. Consequently $e$ acts as an identity on $G$.

Again let $\mu \in \Gamma, x \in$ carrier $\mu, z \in$ carrier $\mu^{-1}$; then $z g \subset$ carrier $\mu^{-1}$ by 4.1, so $x z g \subset($ carrier $\mu)\left(\right.$ carrier $\left.\mu^{-1}\right) \subset g$, and thus $g=(x z g) g=x z g$. Consequently there is a $y$ in $z g$ for which $x y=e$ and $G$ is a group. Moreover $x^{-1}=y \in z g$ so $z \in x^{-1} g$; since $z$ was any element of carrier $\mu^{-1}$, carrier $\mu^{-1} \subset x^{-1} g=y g \subset z g \subset\left(\right.$ carrier $\left.\mu^{-1}\right) g \subset$ carrier $\mu^{-1}$. Thus carrier $\mu^{-1}$ $=z g$ for any $z \in$ carrier $\mu^{-1}$, or carrier $\mu=x g$, for any $x$ in carrier $\mu$, and carrier $\mu$ is a coset of $g$ in $G$. Now

$$
\int f(z) \mu(d z)=\iint f(x y) \eta(d x) \mu(d y), \quad f \in C(S),
$$

since $\mu=\eta \mu$. Since $y \rightarrow \int f(x y) \eta(d x)$ is constant on carrier $\mu$,

$$
\int f(z) \mu(d z)=\int f(x y) \eta(d x)
$$

for any $y$ in carrier $\mu$. Thus $\mu$ is exactly the translate to $y g$ of $\eta$.

Finally suppose $\Gamma$ is closed. If $x \in G^{-}$we can find nets $\left\{x_{\delta}\right\}$ and $\left\{\mu_{\delta}\right\}$ for which $x_{\delta} \rightarrow x, x_{\delta} \in$ carrier $\mu_{\delta}, \mu_{\delta} \in \Gamma$ and $\mu_{\delta} \rightarrow \mu \in \Gamma$. If $x \notin$ carrier $\mu$ then $x g \cap$ carrier $\mu=\phi$ and there is an $f$ in $C(S), 0 \leqq f \leqq 1$, which is 1 on $x g$ and 0 on carrier $\mu$. Since

$$
y \rightarrow \int f(y z) \eta(d z)
$$

is continuous by 1.2 , and assumes the value 1 for $y$ in $x g$, we have

$$
\frac{1}{2} \leqq \int f\left(x_{\delta} z\right) \eta(d z)=\int f(z) \mu_{\delta}(d z) \quad \text { for } \delta \geqq \delta_{0},
$$

despite the fact that 


$$
\int f(z) \mu_{\delta}(d z) \rightarrow \int f(z) \mu(d z)=0 .
$$

Thus $x \in$ carrier $\mu \subset G$, and $G$ is closed, completing our proof.

Actually we can obtain all of the analogous result (Th. 2.3) of [3]; it is easy to see that if $\Gamma$ is closed (as [3] required) then the weak* closed convex hull $\mathscr{C}(\Gamma)$ of $\Gamma$ is the image of $(G / g)^{\sim}$, using exactly the map $T_{\eta}$ of $[3,2.3]$ (alternatively we could note that our measures all lie on a compact topological group $G$, and apply 2.3 of [3]).

THEOREM 4.4. Let $\Sigma$ be a closed subsemigroup of $\widetilde{S}$ with least ideal $\mathscr{I} ;$ let $^{2} S_{1}=\left(\bigcup_{\mu \in \Sigma} \text { carrier } \mu\right)^{-}$, with least ideal I. Then $I=\mathrm{U}_{\mu \in \mathscr{F}}$ carrier $\mu$.

Proof. Since $\mathscr{I}$ is a closed subsemigroup of $\Sigma$, and thus of $\widetilde{S}$, by 4.3, $G=\bigcup_{\mu \in \mathscr{F}}$ carrier $\mu$ is a closed subgroup of $S$, and thus of $S_{1}$. Let $S_{0}=\bigcup_{\mu \in \Sigma}$ carrier $\mu$, and algebraic subsemigroup of $S$ with $S_{0}^{-}=S_{1}$.

Suppose $x S_{1}$ does not contain $G$ for some $x$ in $S_{1}$. Then since $y \in x S_{1} \cap G$ implies $G=y G \subset x S_{1} G \subset x S_{1}, x S_{1} \cap G=\phi$. Consequently there is an $f$ in $C(S)$ which vanishes on $x S_{1}$ and is 1 on $G$. Since $x \in S_{1}=S_{0}^{-}$, there is a net $x_{\delta} \rightarrow x, x_{\delta} \in$ carrier $\mu_{\delta}, \mu_{\delta} \in \Sigma$. For $\nu$ in $\mathscr{I}$, $x$ carrier $\nu \subset x S_{1}$, so that $\int f(x y) \nu(d y)=0$, and therefore $\int f\left(x_{\delta} y\right) \nu(d y) \rightarrow 0$ by 1.2. On the other hand $\mu_{\delta} \nu \in \mathscr{F}$ so that $x_{\delta}$ carrier $\nu \subset$ carrier $\mu_{\delta} \nu$ $\subset G$, and $\int f\left(x_{\delta} y\right) \nu(d y)=1$, a contradiction, whence we conclude that $G \subset x S_{1}$ for all $x$ in $S_{1}$. Thus $G \subset I=\bigcap_{x \in S_{1}} x S_{1}$.

Now for $x$ in $S_{0}$ and $\nu$ in $\mathscr{I}$, the fact that $x$ carrier $\nu \subset G$ shows $x G \subset G$; for $y$ in $G$ then $x y \in G$ for all $x$ in $S_{1}$ since $G$ is closed and $x \rightarrow x y$ continuous. Consequently $x G \subset G$, all $x$ in $S_{1}$, and $G$ is an ideal in $S_{1}$; of course $G$ must then contain the least ideal $I$, whence $G=I$ and our proof is complete.

By virtue of 4.4 and the remark immediately preceding it we obtain, by exactly the proof of $[3,3.2]$,

TheOREM 4.5. Let $\mu \in \widetilde{S}$. Then $(1 / N) \sum_{n=1}^{N} \mu^{n} \rightarrow$ Haar measure on the least ideal of the closed subsemigroup of $S$ generated by carrier $\mu$.

For the proofs of some of our next remarks (and for definitions of the basic entities involved) the reader is referred to [2]. Let $G$ be a locally compact abelian group. Then the weakly almost periodic functions on $G$ form a closed translation invariant subalgebra $W(G)$ of $C(G)$ containing $C_{0}(G)$. Moreover $W(G)$ is isometrically isomorphic to $C\left(G^{w}\right)$, where $G^{w}$ is a compact abelian separately continuous semigroup, the

${ }^{2}$ Separate continuity (applied twice) is sufficient to guarantee that the closure of an :algebraic subsemigroup is a subsemigroup. 
weakly almost periodic compactification of $G$, in which $G$ forms (topologically $^{3}$ and algebraically) a dense open subgroup; the elements in $W(G)$ are just the restrictions, to $G$, of the elements of $C\left(G^{w}\right) . \quad\left(G^{w}\right.$ is not jointly continuous, or a group, unless $G$ is compact.) Naturally each finite measure $\mu$ on $G$ induces an element $\mu^{\prime}$ of $C\left(G^{w}\right)^{*}$, and $\mu \rightarrow \mu^{\prime}$ is easily seen to preserve convolution, norm and order; in particular $\mu \geqq 0$, $\|\mu\|=1$ imply $\mu^{\prime} \in \widetilde{G}^{w}$. If we define the carrier, in $G$, of such a nonnegative $\mu$ to be the closed complement of the union of all open sets of $\mu$-measure zero, then carrier $\mu^{\prime}$ in $G^{w}$ contains the carrier of $\mu$ (since open sets in $G$ remain open in $G^{w}$, and $C_{0}(G) \subset W(G)$ ). Finally let the translate $R_{g} f$ of $f$ be defined by $R_{g} f\left(g^{\prime}\right)=f\left(g^{\prime} g\right), g, g^{\prime}$ in $G, f$ in $W(G)$. We need only apply 4.5 to $S=G^{w}$ and $\mu^{\prime}$ to obtain

THEOREM 4.6. Let $G$ be a locally compact abelian group, and let $\mu \geqq 0$ be an element of $C_{0}(G)^{*}$ of norm 1 . Then there is a non-negative functional $F$ of norm 1 on $W(G)$ for which

$$
\frac{1}{N} \sum_{n=1}^{N} \int_{\theta} f(g) \mu^{n}(d g) \rightarrow F(f),
$$$$
f \text { in } W(G) \text {, }
$$

and $F\left(R_{g} f\right)=F(f)$ for all $g$ in the carrier of $\mu$.

Here $\mu^{n}$ is, of course, the ordinary $n$-fold convolve of $\mu$. As the reader will observe, a related result can be obtained when $G$ is merely. an abelian topological semigroup, as in [2].

Familiar results from ergodic theory suggest an alternative approach. to 4.6, but yield a result of a different nature. Indeed if we define $\mu^{n} * f$, for $f$ in $W(G)$, by $\mu^{n} * f(g)=\int f\left(g g^{\prime}\right) \mu^{n}\left(d g^{\prime}\right)$ then $\mu^{n} * f$ lies in the weakly compact closed convex hull $K$ of the set of translates of $f$, and ergodic theory shows $(1 / N) \sum_{n=1}^{N} \mu^{n} * f$ converges strongly to an $f_{1}$ in $K$ with $\mu * f_{1}=f_{1}$. From this alone it is not all apparent that $f_{1}$ should have the stronger invariance property that $R_{g} f_{1}=f_{1}$ for $g$ in the carrier: of $\mu$. But since $\mu^{n} * f(g)=\int R_{g} f\left(g^{\prime}\right) \mu^{n}\left(d g^{\prime}\right), 4.6$ shows

$$
\frac{1}{N} \sum_{n=1}^{N} \mu^{n} * f(g) \rightarrow F\left(R_{g} f\right)
$$

and $f_{1}(g)=F\left(R_{g} f\right)$, so $f_{1}$ does indeed have the invariance property.. Consequently we have proved

COROLlary 4.7. Let $G$ be a locally compact abelian group, $\mu$ a non-negative measure of norm 1 on $G$. Then the operators

${ }^{3}$ In the more general context of [2] $G$ is only imbedded continuously in $G^{w}$; here $C_{0}(G) \subset W(G)$ guarantees the imbedding is open as well. 


$$
f \rightarrow \frac{1}{N} \sum_{n=1}^{N} \mu^{n} * f
$$

on $W(G)$ converge in the strong operator topology to a projection onto the manifold of functions left fixed by $\left\{R_{g}: g\right.$ in the carrier of $\left.\mu\right\}$.

4.8 REMARK. The remaining result of $\S 3$ of [3], 3.5, extends to the present context with no change in proof; beyond this point, however, there are difficulties in obtaining extensions. In particular $\S 4$ makes strong use of the now lacking property that the closure of an algebraic subgroup of $\widetilde{S}$ be a group.

5. For $E \subset C(X)$ let $\sigma\left(C_{0}(X)^{*}, E\right)$ denote the least fine topology for which the maps

$$
\mu \rightarrow \int f(x) \mu(d x)
$$$$
f \in E
$$

are continuous. When $X$ is taken to be a locally compact abelian group $G, 1.1$ can be applied to some topologies on $C_{0}(G)^{*}$ by virtue of the Fourier-Stieltjes transformation. Let $G^{\wedge}$ denote the character group of $G, \hat{\mu}$ the Fourier-Stieltjes transform of $\mu \in C_{0}(G)^{*}, C_{0}(G)^{* \wedge}$ the set of all such transforms.

THEOREM 5.1. Let $K \subset C_{0}(G)^{*}$ have a uniformly bounded set of Fourier-Stieltjes transforms. Then $K$ is $\sigma\left(C_{0}(G)^{*}, C_{0}\left(G^{\wedge}\right)^{* \wedge}\right)$ compact if (and of course only if ) $K$ is $\sigma\left(C_{0}(G)^{*}, G^{\wedge}\right)=\sigma\left(C_{0}(G)^{*}, P\left(G^{\wedge}\right)^{\wedge}\right)$ compact, where $P\left(G^{\wedge}\right)$ is the set of point masses on $G^{\wedge}$. Moreover $K$ is then weak* compact if bounded.

We need only note that by virtue of the identity

$$
\int_{G} \hat{\nu}\left(g^{-1}\right) \mu(d g)=\int_{G^{\wedge}} \hat{\mu}(\hat{g}) \nu(d \hat{g})
$$

(for $\left.\mu \in C_{0}(G)^{*}, \nu \in C_{0}\left(G^{\wedge}\right)^{*}\right), \sigma\left(C_{0}(G)^{*}, C_{0}\left(G^{\wedge}\right)^{* \wedge}\right.$ ) is the topology $\sigma\left(C_{0}(G)^{* \wedge}\right.$, $C_{0}\left(G^{\wedge}\right)^{*}$ ) (or the weak ${ }^{*}$ topology of $C_{0}\left(G^{\wedge}\right)^{* *}$ ) transported to $C_{0}(G)^{*}$, while $\sigma\left(C_{0}(G)^{*}, P\left(G^{\wedge}\right)^{\wedge}\right)$ corresponds in the same way to $\sigma\left(C_{0}(G)^{* \wedge}, P\left(G^{\wedge}\right)\right.$ ) (or the topology of pointwise convergence). Thus 1.1 can be applied. For the final statement, note that $C_{0}\left(G^{\wedge}\right)^{* \wedge}$ contains $L_{1}\left(G^{\wedge}\right)^{\wedge}$, which defines the weak* topology on bounded subsets of $C_{0}(G)^{*}$.

\section{REFERENCES}

O. R. C. Buck, Bounded continuous functions on a locally compact space, Michigan J., 5 (1958), 95-104.

1. R. Ellis, Locally compact transformation groups, Duke Math. J., 24 (1957), 119-126.

2. K. de Leeuw and I. Glicksberg, Applications of almost periodic compactifications, to appear. 
3. I. Glicksberg, Convolution semigroups of measures, Pacific. J. Math., 9 (1959), 51-67.

4. I. Glicksberg, Uniform boundedness for groups, to appear.

5. A. Grothendieck, Sur la complétion du dual d'un espace localement convexe, Comptes: Rendues, 230 (1950), 605-606.

6. A. Grothendieck, Critères de compacité dans les espaces fonctionnels généraux, Amer. J. Math., 74 (1952), 168-186.

7. H. Hahn, Reele Functionen I, Leipzig, 1932.

UNIVERSITY OF NOTRE DAME 


\section{PACIFIC JOURNAL OF MATHEMATICS}

\section{EDITORS}

Ralph S. Phillips

Stanford University

Stanford, California

F. H. BrownelL

University of Washington

Seattle 5 , Washington
A. L. Whiteman

University of Southern California Los Angeles 7. California

L. J. PAIGE

University of California

Los Angeles 24, California

ASSOCIATE EDITORS
E. F. BECKENBACH
D. DERRY
H. L. ROYDEN
E. G. STRAUS
T. M. CHERRY
M. OHTSUKA
E. SPANIER
F. WOLF

\section{SUPPORTING INSTITUTIONS}

UNIVERSITY OF BRITISH COLUMBIA

CALIFORNIA INSTITUTE OF TECHNOLOGY

UNIVERSITY OF CALIFORNIA

MONTANA STATE UNIVERSITY

UNIVERSITY OF NEVADA

NEW MEXICO STATE UNIVERSITY

OREGON STATE COLLEGE

UNIVERSITY OF OREGON

OSAKA UNIVERSITY

UNIVERSITY OF SOUTHERN CALIFORNIA
STANFORD UNIVERSITY

UNIVERSITY OF TOKYO

UNIVERSITY OF UTAH

WASHINGTON STATE COLLEGE,

UNIVERSITY OF WASHINGTON

AMERICAN MATHEMATICAL SOCIETY

CALIFORNIA RESEARCH CORPORATION

HUGHES AIRCRAFT COMPANY

SPACE TECHNOLOGY LABORATORIES

NAVAL ORDNANCE TEST STATION

Printed in Japan by International Academic Printing Co., Ltd., Tokyo, Japan

Reprinted 1966 in the United States of America 


\section{Pacific Journal of Mathematics}

\section{Vol. 11, No. $1 \quad$ November, 1961}

A. A. Albert, Generalized twisted fields ............................ 1

Richard Arens, Operational calculus of linear relations ................... 9

John Herbert Barrett, Disconjugacy of a self-adjoint differential equation of the fourth order ....................................... 25

Paul Richard Beesack, Hardy's inequality and its extensions ............... 39

Julius Rubin Blum and David Lee Hanson, On invariant probability measures.

II .............................................

Robert Allen Bonic, Symmetry in group algebras of discrete groups.......... 73

R. Creighton Buck, Multiplication operators ...................... 95

Jack Gary Ceder, Some generalizations of metric spaces ................. 105

Meyer Dwass, Random crossings of cumulative distribution functions ......... 127

Albert Edrei, Wolfgang H. J. Fuchs and Simon Hellerstein, Radial distribution and

deficiencies of the values of a meromorphic function ............... 135

William Cassidy Fox, Harmonic functions with arbitrary local singularities ..... 153

Theodore Thomas Frankel, Manifolds with positive curvature ............... 165

Avner Friedman, A strong maximum principle for weakly subparabolic

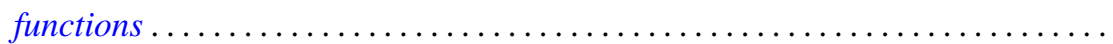

Watson Bryan Fulks and J. O. Sather, Asymptotics. II. Laplace's method for

multiple integrals ......................................

Adriano Mario Garsia and Eugene Richard Rodemich, An embedding of Riemann

surfaces of genus one ..................................... 193

Irving Leonard Glicksberg, Weak compactness and separate continuity......... 205

Branko Grünbaum, On a conjecture of H. Hadwiger .................. 215

Frank J. Hahn, On the action of a locally compact group on $E_{n} \ldots \ldots \ldots \ldots \ldots . . \ldots 221$

Magnus R. Hestenes, Relative hermitian matrices ..................... 225

G. K. Kalisch, On similarity invariants of certain operators in $L_{p} \ldots \ldots \ldots \ldots .247$

Yitzhak Katznelson and Walter Rudin, The Stone-Weierstrass property in Banach

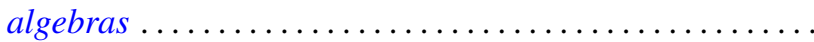

Samir A. Khabbaz, The subgroups of a divisible group $G$ which can be represented as intersections of divisible subgroups of $G \ldots \ldots \ldots \ldots \ldots \ldots \ldots \ldots \ldots . \ldots \ldots 7$

Marvin Isadore Knopp, Construction of a class of modular functions and forms .......................................... 275

Charles Alan McCarthy, Commuting Boolean algebras of projections .......... 295

T. M. MacRobert, Transformations of series of E-functions ................ 309

Heinz Renggli, An inequality for logarithmic capacities ................. 313

M. S. Robertson, Applications of the subordination principle to univalent functions .......................................... 315

David Sachs, Partition and modulated lattices ..................... 325

Frank S. Scalora, Abstract martingale convergence theorems ............... 347

Elbert A. Walker, Torsion endomorphic images of mixed Abelian groups ........ 375

Morgan Ward, The prime divisors of Fibonacci numbers................. 379

Charles R. B. Wright, On the nilpotency class of a group of exponent four....... 387 